\title{
OFFLINE SIGNATURE VERIFICATION USING NEURAL NETWORK
}

\section{DHOTRE S.R.}

Dept. of Computer Science \& Engineering, Gogte Institute of Technology, Belgaum.

*Corresponding Author: Email- srdhotre@rediffmail.com

Received: April 12, 2012; Accepted: May 15, 2012

\begin{abstract}
This paper deals with the offline signature verification system that is useful in automatic identification of signature forgeries involved in the bank, revenue and various other government documents. A set of features is extracted from the scanned image of the given sample of signature which is then applied to a neural network classifier to classify the sample into genuine, skilled forgery, unskilled forgery or random forgery. Artificially generated genuine and forgery samples from enrollment reference signatures are used to train the network, which allows definite training control and at the same time significantly reduces the number of enrollment samples required to achieve a good performance. The performance of such a verification system can be measured by deriving the values of two important performance indices. The false acceptance rate (FAR) is accepting forgery signature thinking it is a genuine signature and false rejection rate (FRR) which is rejecting the genuine signature thinking it is a forgery signature. The performance of the system is mainly influenced by the type of features chosen and the classifier that is selected. The Global features that are extracted from every pixel that lies within a rectangle circumscribing the signature, provide information about the specific cases of the signature shape. The Local or Grid features that are derived from the distribution of pixels over the segments or limited regions of the signature provide overall signature appearance information. Various classifiers have been tried in the literature; the important among them are Neural Network classifier, Fuzzy inference system, Baye's classifier, Support Vector Machine etc. In the proposed system, a combination of global, and grid features are extracted from signature images for classifying them using neural network classifier based on back-propagation learning algorithm .
\end{abstract}

Keywords- offline signature verification, feature extraction, neural network classifier.

Citation: Dhotre S.R., et al. (2012) Offline Signature Verification using Neural Network. International Journal of Machine Intelligence, ISSN: 0975-2927 \& E-ISSN: 0975-9166, Volume 4, Issue 1, pp.-408

Copyright: Copyright@2012 Dhotre S.R., et al. This is an open-access article distributed under the terms of the Creative Commons Attribution License, which permits unrestricted use, distribution, and reproduction in any medium, provided the original author and source are credited. 